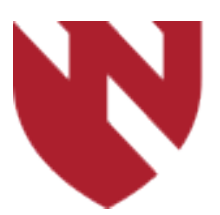

December 2019

\title{
Long Term Outcomes of Surgical and Clinical Symptoms Following Minimally Invasive Heller Myotomy: A Retrospective Clinical Database Review
}

\author{
Laura E. Flores \\ University of Nebraska Medical Center \\ Priscila Rodrigues Armijo \\ University of Nebraska Medical Center \\ Bhavani Pokala \\ University of Nebraska Medical Center \\ Sarah Samreen \\ University of Nebraska Medical Center \\ Dmitry Oleynikov \\ University of Nebraska Medical Center \\ Tell us how you used this information in this short survey. \\ Follow this and additional works at: https://digitalcommons.unmc.edu/gmerj \\ Part of the Digestive System Diseases Commons, Gastroenterology Commons, and the Surgery \\ Commons
}

\section{Recommended Citation}

Flores, L. E., Rodrigues Armijo, P., Pokala, B., Samreen, S., , Oleynikov, D. Long Term Outcomes of Surgical and Clinical Symptoms Following Minimally Invasive Heller Myotomy: A Retrospective Clinical Database Review. Graduate Medical Education Research Journal. 2019 Dec 13; 1(1).

https://digitalcommons.unmc.edu/gmerj/vol1/iss1/6

This Original Report is brought to you for free and open access by DigitalCommons@UNMC. It has been accepted for inclusion in Graduate Medical Education Research Journal by an authorized editor of DigitalCommons@UNMC. For more information, please contact digitalcommons@unmc.edu. 


\title{
Long Term Outcomes of Surgical and Clinical Symptoms Following Minimally Invasive Heller Myotomy: A Retrospective Clinical Database Review
}

\begin{abstract}
I

Introduction

Minimally invasive Heller myotomy rates have increased, but little evidence is available regarding long term clinical and surgical outcomes. Our aim was to evaluate long term symptom improvement and medication resolution for patients undergoing minimally invasive Heller myotomy.
\end{abstract}

Methods

A single-institution database was retrospectively reviewed for patients undergoing laparoscopic Heller myotomy (LHM) or robotic Heller myotomy (RHM) during 2007-2018. Patients with primary HM followed by a Dor fundoplication were included. Demographics and surgical data were analyzed. Esophageal symptoms, testing, and medication use were collected preoperatively (pre-op), at 6-month (6-mo), 12-month (12-mo), and long-term (LT; 12-mo+) follow-up. Analysis was performed using SPSS v.23.0, $\alpha=0.05$.

Results

Eighty eight patients (RHM:N=66; LHM:N=22) were included. The majority were male $(62.5 \%)$ and Caucasian (89.8\%), with a mean BMI of 27.3. Two patients had an intraoperative esophageal perforation, each repaired with a non-eventful postoperative course. Mean follow-up time was 71 months overall, 75 months [11-171 months] and 40 months [6-158 months] for LHM and RHM, respectively. All patients showed significant LT improvement of regurgitation, solid or liquid dysphagia, and Eckardt Score. Postoperative proton pump inhibitor (PPI) use was significantly lower at LT (LHM:31.3\%, RHM:19.4\%) compared to pre-op.

Conclusion

In this study, minimally invasive HM was a safe and effective treatment for achalasia symptom resolution in the long term. Therefore, in our experience, minimally invasive HM is a safe therapy that helps maintain symptom resolution.

\section{Keywords}

Achalasia, Heller myotomy, robotic surgery

\section{Creative Commons License}

(c) (i) $\Theta$

This work is licensed under a Creative Commons Attribution-Noncommercial-No Derivative Works 4.0 License. 


\section{Long Term Outcomes of Surgical and Clinical Symptoms Following Minimally Invasive Heller Myotomy: A Retrospective Clinical Database Review}

Laura E. Flores ${ }^{1}$, Priscila Rodrigues Armijo ${ }^{1}$ Bhavani Pokala ${ }^{1,2}$, Sarah Samreen ${ }^{1,2}$, Dmitry Oleynikov ${ }^{1,2}$

${ }^{1}$ University of Nebraska Medical Center, Center for Advanced Surgical Technology

'University of Nebraska Medical Center, Department of Surgery

https://doi.org/10.32873/unmc.dc.gmerj.1.1.006

\begin{abstract}
Introduction: Minimally invasive Heller myotomy (MIHM) rates have increased, but little evidence is available regarding long term (LT) clinical and surgical outcomes. Our aim was to evaluate LT symptom improvement and medication resolution for patients undergoing MIHM.
\end{abstract}

\begin{abstract}
Methods: A single-institution database was retrospectively reviewed for patients undergoing laparoscopic Heller myotomy (LHM) or robotic Heller myotomy (RHM) during 2007-2018. Patients with primary HM followed by a Dor fundoplication were included. Demographics, surgical data and direct cost were analyzed. Esophageal symptoms, testing, and medication use were collected pre-operatively (pre-op), at 6-month (6-mo), 12-month (12-mo), and LT (15-mo+) follow-up. Analysis was performed using SPSS v.23.0, $\alpha=0.05$.
\end{abstract}

Results: Eighty eight patients (RHM: $\mathrm{N}=66$; LHM:N=22) were included. The majority were male (62.5\%) and Caucasian (89.8\%), with a mean BMI of 27.3. Two patients had an intraoperative esophageal perforation, each repaired with a non-eventful post-operative course. Mean follow-up time was 71 months overall, 75 months [11-171 months] and 40 months [6-158 months] for LHM and RHM, respectively. All patients showed significant LT improvement of regurgitation, solid or liquid dysphagia, and Eckardt Score. Postoperative proton pump inhibitor (PPI) use was significantly lower at LT (LHM:31.3\%, RHM:19.4\%) compared to pre-op.

Conclusion: In this study, MIHM was a safe and effective treatment for achalasia symptom resolution in the LT. Therefore, in our experience, MIHM is a safe therapy that helps maintain symptom resolution.

\section{Introduction}

Achalasia is a functional disorder of the esophageal body and lower esophageal sphincter (LES), resulting in a wide array of clinical symptoms. ${ }^{1,2}$ Although the cause and contributing factors to the development of achalasia are unknown, the resulting symptoms are well detailed and include dysphagia, regurgitation, chest pain and cough. Several treatment options are available for achalasia, but Heller myotomy (HM) is the accepted definitive surgical treatment, due to its recognized long term (LT) benefits in symptom resolution, medication resolution, and quality of life improvements. ${ }^{3,4}$ Since the first HM was performed over 100 years ago, tremendous advancements have been made in the procedure, including a transition away from an open approach to a minimally invasive approach. ${ }^{5}$ Laparoscopic, and more recently, robotic approaches are being adopted because minimally invasive approaches have been shown to decrease post-operative complications and hospital length of stay. ${ }^{6}$ Minimally invasive surgery (MIS) has also been shown to reduce operating time, reduce re-bleeding, and decrease mortality in a wide spectrum of specialties ranging from neurosurgery to orthopaedics. ${ }^{7,8}$ In addition to positive post-operative outcomes, dysphagia rates, the leading sign of successful achalasia treatment, have been shown to decrease significantly after minimally invasive HM (MIHM). ${ }^{9,10}$ This is an important finding because progressive dysphagia leads to an extremely poor quality of life in achalasia patients if left untreated. ${ }^{11}$

Few studies have examined the benefits of short-term and LT outcomes after laparoscopic HM. Other authors have described benefits in regards to lower rates of dysphagia and heartburn, as well as improved swallowing status. ${ }^{12,13}$ Still, fewer authors have looked at robotic $\mathrm{HM}$, with small cohorts ranging from 14-56 patients. ${ }^{14,15}$ Therefore, despite the data that exist on MIS benefits for surgical procedures in general, there is a lack of research substantiating feasibility and LT results after both minimally invasive Heller approaches, especially those with a long follow-up time. Given the efficacy of minimally invasive approaches in other surgical fields, the aim of our study was to evaluate short and LT symptom improvement in patients who underwent MIHM.

\section{Methods}

A single institution database was retrospectively reviewed for patients who underwent either a robotic-assisted (RHM) or laparoscopic Heller myotomy (LHM) between 2007 and 2018. Only adult patients who had a diagnosis of achalasia and who underwent primary HM followed by a Dor fundoplication were included in this study. Three patients underwent Nissen or Toupet fundoplication and were excluded. The technique for HM followed by Dor fundoplication comprises of esophageal mobilization, release of esophageal and gastric muscles fibers, followed by a $180^{\circ}$ anterior fundoplication. The goal of the procedure is to facilitate LES function and resolve achalasia. ${ }^{16}$ All surgeries in this cohort were performed by the same surgeon. Exclusion criteria included any patient with other esophageal disease, such as ineffective dysmotility or malignancy. A diagnosis of achalasia was determined by the Chicago Classification of esophageal dysmotility standards, according to preoperative esophageal manometry testing. ${ }^{17}$

Demographics, such as gender, age, race, body mass index (BMI), and smoking history were collected. Patient health information such as pre-operative esophageal symptoms and testing, medication use, medical history, and pre-operative comorbidities were collected. Pre-operative esophageal testing included esophagogastroduodenoscopy (EGD), barium esophagram, manometry, and $\mathrm{pH}$ testing. Esophageal symptoms were analyzed using a validated esophageal score, ${ }^{18}$ and analysis was based on a composite of mean scores. Surgical data comprised surgical approach, operating time, blood loss, intraoperative complications, and length of stay. Post-operative complications, including bleeding, ileus, and others were recorded. Frequency of esophageal symptoms and Eckardt score were collected using a validated intra and extra esophageal symptom survey pre-operatively (pre-op), and at 6-month (6mo), 12-month (12-mo), and LT (15+ months) follow-up.

Data are presented as frequency for categorical data, or as median or mean, for continuous data according to normality. Descriptive statistics were calculated for the cohort, and comparisons were made using t-tests or chi-square testing. The Sign test with Bonferroni correction was used for additional evaluation of symptoms improvement over time compared to the baseline. ${ }^{19}$ Statistical analysis was performed using SPSS v.25.0, with $\alpha=0.05$. 


\section{Results}

Eighty-eight patients underwent MIHM followed by Dor fundoplication (RHM: $\mathrm{N}=66$; LHM: $\mathrm{N}=22$ ) and met the inclusion criteria for the study. The mean age was $53.1 \pm 17.6$ years and the mean BMI was $27.3 \mathrm{~kg} / \mathrm{m} 2$. Over half of patients were male $(62.5 \%)$ and Caucasian (89.8) (Table 1).

Table 2 shows intra-operative findings. All hiatal hernias were repaired at the time of surgery. A majority of patients had minimal $(<50 \mathrm{~mL})$ blood loss, and two patients had an intra-operative complication. One patient in each group had an intra-operative esophageal perforation, which was repaired with a non-eventful post-operative course. Postoperatively, length of stay was $2.14 \pm 2.98$ days overall, $1.87 \pm 2.68$ days for the robotic approach, and $3.29 \pm 3.9$ for the laparoscopic approach $(\mathrm{p}=0.203)$. Post-operative complications occurred in four patients overall, three with post-operative bleeding, and one with post-operative ileus.

Overall symptom scores improved over time, along with consistent improvement in
Eckardt score, as shown in Table 3. Table 4 demonstrates that symptom improvement from baseline was significant in all symptoms, with dramatic improvement in solid dysphagia from baseline to LT $(\mathrm{p}<0.001)$. Whether patients had laparoscopic or robotic procedure, there were no differences in postoperative symptom scores.

Mean LT follow-up time was 70 months \pm 47.07. Follow up ranged from 11-171 months in our cohort, with over a third of patients having LT follow up data over 4 years $(\mathrm{N}=31)$.

Dilation rates and recurrence of objective GERD on upper gastrointestinal imaging (UGI) were low in this cohort. Overall, 11 patients $(12.5 \%)$ underwent post-operative dilation, with three of them requiring multiple procedures. Of the 11 patients dilated, two had evidence of peptic stricture and responded well to dilation and proton pump inhibitor therapy. In the remaining nine, those with dilated esophagus and angulation were dilated with a $20 \mathrm{~mm}$ balloon. No patients had incomplete myotomy by manometry. The earliest dilation happened at least seven months after the surgery. UGI was performed

\section{Table 1.}

Demographics and pre-operative characteristics of patients undergoing minimally invasive Heller myotomy. Descriptive statistics were calculated.

\begin{tabular}{|c|c|c|c|c|c|c|}
\hline & \multicolumn{2}{|c|}{$\begin{array}{l}\text { Overall } \\
(n=88)\end{array}$} & \multicolumn{2}{|c|}{$\begin{array}{c}\mathrm{RHM} \\
(\mathrm{n}=66)\end{array}$} & \multicolumn{2}{|c|}{$\begin{array}{c}\text { LHM } \\
(n=22)\end{array}$} \\
\hline & $\mathbf{N}$ & $\%$ & $\mathbf{N}$ & $\%$ & $\mathbf{N}$ & $\%$ \\
\hline Female & 33 & 37.5 & 21 & 31.8 & 12 & 54.5 \\
\hline Male & 55 & 62.5 & 45 & 68.2 & 10 & 45.5 \\
\hline Age - years (mean \pm SD) & $53.1 \pm 17.6$ & & $51.4 \pm 16.5$ & & $57.9 \pm 19.8$ & \\
\hline \multicolumn{7}{|l|}{ Race } \\
\hline Caucasian & 79 & 89.8 & 57 & 86.4 & 22 & 100 \\
\hline AA & 3 & 3.4 & 3 & 4.5 & 0 & 0 \\
\hline Other & 3 & 3.4 & 3 & 4.5 & 0 & 0 \\
\hline Pre-op BMI kg/m² & $27.3 \pm 4.6$ & & $27.2 \pm 4.6$ & & $28.5 \pm 3.6$ & \\
\hline Pre-op Eckardt Score & $4.4 \pm 2.5$ & & $4.2 \pm 2.8$ & & $4.4 \pm 2.8$ & \\
\hline Overall Follow-up (months) & $70.1 \pm 47.0$ & & $40(6-158)$ & & $75(11-171)$ & \\
\hline
\end{tabular}

RHM Robotic-assisted Heller Myotomy; LHM Laparoscopic Heller Myotomy; Pre-op preoperative; AA African American

\section{Table 2.}

Intra-operative and peri-operative data on patients undergoing minimally invasive Heller myotomy. Comparisons between groups were made using Chi-square analysis or Wilcoxon rank sum test.

\begin{tabular}{rcccc} 
& Overall & RHM & LHM & p value \\
\hline Operative Hiatal Hernia Present & $10(11.4 \%)$ & $7(10.6 \%)$ & $3(13.6 \%)$ & 0.521 \\
\hline OR Time $(\boldsymbol{m i n})$ & $190 \pm 48.32$ & $193.0 \pm 48.4$ & $175.2 \pm 48.1$ & 0.931 \\
\hline Blood Loss $(\mathbf{m l})$ & $18.83 \pm 22.30$ & $15.6 \pm 19.2$ & $30.56 \pm 29.7$ & 0.203 \\
\hline LOS & $2.14 \pm 2.98$ & $1.78 \pm 2.5$ & $3.29 \pm 3.9$ & 0.059 \\
\hline
\end{tabular}

RHM Robotic-assisted Heller Myotomy; LHM Laparoscopic Heller Myotomy; LOS Length of Stay

at one year in $57(65 \%)$ patients. Only three patients were found to have objective GERD diagnosed on post-operative UGI (5.2\%), whereas two patients had asymptomatic recurrence of hiatal hernia. Use of postoperative PPI was also reduced to just $23 \%$ of patients reporting PPI use, compared to $49 \%$ at baseline (Table 5).

\section{Discussion}

This study demonstrates the safety and efficacy of MIHM. Our cohort of 88 patients had a less than $4.5 \%$ complication rate, with low rates of post-operative dilation. A significant number of patients reported improvement symptoms and heartburn scores in the LT. Furthermore, over $50 \%$ of the patients who reported pre-operative PPI use were able to stop taking these medications post-operatively.

The demographics of our cohort, including race, gender, BMI, and age are similar to those reported in the current HM literature. ${ }^{16,20,21}$ Conversely, our mean operative time of 190 minutes was slightly longer than that reported in the literature (range:89-141 min). ${ }^{20,22}$ In the current study, data were collected from an academic medical center where residents and students often play a role in the operation. This may have contributed to the increased length of OR time. Our average length of stay was 2.14 days which falls within the expected range of 1-4.3 days..$^{19,22}$

The reported overall complication rates for MIHM range from 1.8-6.3\% ${ }^{20,21}$ Esophageal perforation is considered to be the most prevalent and serious complication of MIHM. In our study, only two patients $(2.3 \%)$ suffered esophageal perforations. In both of these instances, perforations were repaired intraoperatively and patients had an otherwise unremarkable post-operative course. This is within the reported perforation rates in the literature ranging from $1-13 \%$ with one study citing the esophageal perforation rate as high as $33 \%{ }^{23}$ Mortality rates following perforation have been reported to be as high as $40 \% .{ }^{24-27}$ The low rate of perforations in our cohort, coupled with a significant improvement in esophageal symptom scores, supports that MIHM is a safe and efficacious treatment for achalasia.

An important marker of success following surgical treatment of achalasia is the resolution of dysphagia and other esophageal symptoms..${ }^{90}$ Our study demonstrated that MIHM provided resolution of esophageal symptoms, including dysphagia, heartburn, regurgitation, and cough, evaluated at LT. In a LT study by Jeansonne et al., which evaluated 
esophageal symptoms, $95 \%$ of patients who underwent MIHM demonstrated satisfaction with their operation, over a follow-up period of 10 years. ${ }^{12}$ Patients reported symptom improvement in dysphagia and cough at shortand LT follow-up, when compared to baseline $(p<0.05)$. Improvement was not significant at LT follow-up in other esophageal symptoms such as heartburn, chest pain, regurgitation or asthma ( $p>0.05)$. At 10-year follow-up, $62.5 \%$ of patients reported a lower symptom score for dysphagia when compared to the baseline. Whereas in our study, $98 \%$ of our patients reported improved dysphagia rates at LT, compared to baseline. Although Jeansonne et al. indicated the effectiveness of MIHM given the robust LT symptom resolution rates, their study was limited by a sample size of only 17 patients. ${ }^{12}$ The difference in sample size between the two studies ( $\mathrm{N}=88$ vs. $\mathrm{N}=17$ ) may have contributed to the variable rates of symptom resolution; however, both studies ultimately corroborate the efficacy of MIHM.

In a study of over 200 patients, Krishnamohan. et al., evaluated the severity and frequency of dysphagia of patients after MIHM using validated questionnaires. ${ }^{13}$ The questionnaires were able to capture robust data on dysphagia variability. The authors stated a significant decrease in dysphagia postoperatively, with a third of patients $(\mathrm{N}=80)$ reporting complete resolution of dysphagia at 6.4 years follow-up. Additionally, swallowing difficulty was further classified into dysphagia for liquids $31 \%(50 / 161)$ or solids $92.5 \%$ (149/161). These results were much higher than our LT dysphagia rates, which could be due to the variation in surgery received by the patients. In some cases, no fundoplication was performed $(\mathrm{N}=23 ; 4.6 \%)$. The surgeon's preference for the type of fundoplication also varied between Toupet $(\mathrm{N}=268)$ and Dor $(\mathrm{N}=209)$.

Our patients demonstrated no difference between LHM and RHM in symptom resolution. Similar results are seen when both minimally invasive procedures, laparoscopy and robotic-assisted, were evaluated independently. A 2004 study by Ruurda et al, sought to gather data on symptom resolution for a small group of 14 patients undergoing robotic HM (RHM). They found that $86 \%$ $(12 / 14)$ of patients reported dysphagia relief after the procedure, whereas $14 \%(2 / 14)$ reported heartburn, with a mean follow-up time of only 11 months. Another study of 73 patients who underwent RHM, showed that $96 \%$ reported their swallowing status to be good/excellent, with little information about other symptom resolution. ${ }^{28}$

Table 3.

Overall mean symptom scores over time for patients who underwent minimally invasive Heller myotomy.

\begin{tabular}{rcccc} 
& Pre-op & 6-mo & 12-mo & LT \\
\hline Heartburn & 1.631 & 0.360 & 0.412 & 0 \\
\hline Regurgitation & 2.594 & 0.230 & 0.118 & 0 \\
\hline Solid Dysphagia & 3.452 & 0.633 & 0.500 & 0.02 \\
\hline Liquid Dysphagia & 2.833 & 0.298 & 0.194 & 0 \\
\hline Eckardt Score & 4.398 & 0.760 & 0.560 & 0.315 \\
\hline
\end{tabular}

Pre-op preoperative; 6-mo 6 months follow-up; 12-mo 12 months follow-up; $L T$ Long-term follow-up

\section{Table 4.}

Symptom score improvement over time in patients who underwent minimally invasive Heller myotomy. Repeated measures ANOVA was used to test for symptom score improvement.

\begin{tabular}{rccccccc} 
& \multicolumn{2}{c}{$\mathbf{6 m o}(\mathbf{n}=\mathbf{4 3})$} & \multicolumn{2}{c}{$\mathbf{1 2 m o}(\mathbf{n}=\mathbf{4 4})$} & \multicolumn{2}{c}{$\mathbf{L T}(\mathbf{n = 5 4})$} \\
\hline Symptom & \%Improved & $\mathbf{p}$ & \%lmproved & $\mathbf{p}$ & \%lmproved & $\mathbf{p}$ \\
\hline Heartburn & 54 & $<0.001$ & 53 & $<0.001$ & 61 & $<0.001$ \\
\hline Regurgitation & 78 & $<0.001$ & 80 & $<0.001$ & 79 & $<0.001$ \\
\hline Solid Dysphagia & 93 & $<0.001$ & 77 & $<0.001$ & 98 & $<0.001$ \\
\hline Liquid Dysphagia & 90 & $<0.001$ & 89 & $<0.001$ & 84 & $<0.001$ \\
\hline Any Dysphagia & 62 & $<0.001$ & 70 & $<0.001$ & 96 & $<0.001$ \\
\hline
\end{tabular}

Table 5.

Long term proton pump inhibitor resolution in patients who underwent minimally invasive Heller myotomy.

\begin{tabular}{rccccccccc} 
& \multicolumn{2}{c}{ Pre-op } & \multicolumn{2}{c}{ 6-mo } & \multicolumn{2}{c}{ 12-mo } & \multicolumn{2}{c}{ LT } \\
\hline PPI use & $\mathrm{N}$ & $\%$ & $\mathrm{~N}$ & $\%$ & $\mathrm{~N}$ & $\%$ & $\mathrm{~N}$ & $\%$ \\
\hline Overall & 36 & 49 & 10 & 18 & 1 & 2.8 & 11 & 23 \\
\hline LHM & 11 & 61 & 3 & 19 & 0 & 0 & 5 & 31 \\
\hline RHM & 25 & 45 & 7 & 18 & 1 & 3.8 & 6 & 19 \\
\hline
\end{tabular}

Preop preoperative; 6-mo 6 months follow-up; 12-mo 12 months follow-up; LT Long-term follow-up, RHM Robotic-assisted Heller Myotomy; LHM Laparoscopic Heller Myotomy

Although HM is the gold standard treatment for achalasia, new alternatives have emerged including per oral endoscopic myotomy (POEM). POEM is a natural orifice intervention for achalasia, showing mixed results in the short and LT compared to HM. ${ }^{29,30}$ POEM is praised for its short term efficacy, low post-operative pain scores, and quick return to normal function. However, LT efficacy of POEM remains debated. ${ }^{31,32} \mathrm{~A}$ systematic review comparing POEM to HM in the LT, noted that POEM has a much higher incidence of pathological reflux, which could potentially lead to complications such as Barrett's esophagus. ${ }^{30}$ Additionally, one series noted the high incidence of intraoperative complications including burns and small perforations (21\%) and symptomatic capnoperitoneum (11\%). ${ }^{33}$ This study also echoed the high incidence of GERD following POEM, with a reported $38 \%$ of their patient population experiencing GERD. Although our focus was not to compare POEM to HM, our objectively measured GERD rates were much lower at $5.2 \%$ following HM, when compared to POEM.

Freedom from medication after surgery is an additional important marker of success in these patients. We found that objectively measured reflux in our patient population was $5.7 \%$ at 1 -year follow-up. PPI use at 1-year follow-up was $2.8 \%$, which is lower than our UGI objectively measured GERD. At LT follow-up 23\% of our cohort reported PPI use. This is much lower than reported rates in the literature. Medication use rates after MIHM has been reported to range from $25 \%$ to $66 \%$ of patients taking anti-reflux medications such as antacids, Histamine H2-receptor antagonists (H2 blockers), calcium channel blockers (CCB) or PPI post-operatively. ${ }^{34,35}$ 
A study of over 500 patients who underwent MIHM showed $66 \%$ of patients $(159 / 241)$ were taking antacids, Histamine 2 blockers, or PPI at LT follow-up (mean 6.4 years). ${ }^{13}$ While these authors used objective testing in the form of pre-operative manometry to diagnose achalasia, there was no objective testing routinely obtained post-operatively. In a different cohort, $25 \%$ of patients had severe enough reflux symptoms post operatively to require PPI therapy. ${ }^{35}$

Perry et al., evaluated the difference in medication use between MIS approaches, and found $56 \%$ of robotic patients and $80 \%$ of laparoscopic patients required medication for reflux at LT follow-up. ${ }^{14}$ While they cite their PPI use as "very high," the authors point to a need for objective testing to determine if the symptoms are truly a result of acid reflux. Others have reported more descriptive breakdowns, with $41 \%, 7 \%$ and $2 \%$ of patients post-operatively taking PPIs, CCBs, and Nitroglycerine, respectively. ${ }^{36}$ In our

\section{References}

1 Boeckxstaens GEE. Achalasia. Best Practice \& Research Clinical Gastroenterology. 2007;21(4):595608.

2 Schlottmann F, Neto RML, Herbella FAM, et al. Esophageal Achalasia: Pathophysiology, Clinical Presentation, and Diagnostic Evaluation. The American Surgeon. 2018;84(4):467-472.

3 SSAT patient care guidelines. Esophageal achalasia. Journal of gastrointestinal surgery: official journal of the Society for Surgery of the Alimentary Tract. 2007;11(9):1210-1212.

4 Patti MG, Fisichella PM, Perretta S, et al. Impact of minimally invasive surgery on the treatment of esophageal achalasia: a decade of change. Journal of the American College of Surgeons. 2003;196(5):698703; discussion 703-695.

5 Payne WS. Heller's contribution to the surgical treatment of achalasia of the esophagus. 1914. The Annals of thoracic surgery. 1989;48(6):876-881.

6 Schuchert MJ, Luketich JD, Landreneau RJ, et al. Minimally-invasive esophagomyotomy in 200 consecutive patients: factors influencing postoperative outcomes. The Annals of thoracic surgery. 2008;85(5):1729-1734.

$7 \mathrm{Ng} \mathrm{KKM}$, Cheung JPY. Is minimally invasive surgery superior to open surgery for treatment of lumbar spinal stenosis? A systematic review. Journal of orthopaedic surgery (Hong Kong). 2017;25(2):2309499017716254.

8 Xia Z, Wu X, Li J, et al. Minimally Invasive Surgery is Superior to Conventional Craniotomy in Patients with Spontaneous Supratentorial Intracerebral Hemorrhage: A Systematic Review and Meta-Analysis. World neurosurgery. 2018;115:266-273.

9 Heniford BT, Matthews BD, Kercher KW, et al. Laparoscopic anterior esophageal myotomy and toupet fundoplication for achalasia. The American surgeon. 2001;67(11):1059-1065; discussion 1065-1057.

10 Laurino-Neto RM, Herbella F, Schlottmann F, et al. Evaluation of esophageal achalasia: from symptoms to the chicago classification. Arquivos brasileiros de cirurgia digestiva $: A B C D=$ Brazilian archives of digestive surgery. 2018;31(2): $1376-\mathrm{e} 1376$. patient cohort, only $23 \%$ of patients reported taking PPIs at LT follow-up, versus $49 \%$ at pre-op.

Our study has several limitations, including its retrospective nature and the limited sample size. However, this is one of the largest studies to our knowledge and with an overall response rate of $61 \%(54 / 88)$ at LT followup, we found consistent data collection in our patient population. In order to limit the effects of recall bias, we routinely collected symptom scores at 3-, 6- and 12-months post-operatively and established these time points as our standard of care. The survey we provided pre- and post-operatively at intervals of 3-, 6-, 12-month, and LT, is a significant assessment of a spectrum of esophageal and extra-esophageal symptoms, and has been validated in previous publications. ${ }^{19}$ Further, the survey is a good measurement tool for reported symptoms and is clinically relevant to our patient population. Additionally, we have pertinent information on reported dysphagia, which is the most important symptom relating to esophageal dysmotility, and our survey gathers robust data on dysphagia resolution. Due to the long duration of follow-up in this study, patients who were categorized as "lost to follow-up" were elderly and died of unrelated causes. LT objective measurement should be collected in order to determine if the PPI use is related to symptomatic GERD, or prescribed by another specialty due to reasons unrelated to GERD. ${ }^{37}$

In conclusion, this study demonstrates the efficacy of MIHM in symptom relief and medication freedom for patients with achalasia. MIHM provides significant symptom relief in both the short-term and LT along with significant reduction in use of anti-reflux medications. Given the feasibility and safety of both laparoscopy and the robotic platform for a MIHM, either approach can be used and should be based on surgeon preference.
11 Khajanchee YS, Kanneganti S, Leatherwood AE, Hansen PD, Swanstrom LL. Laparoscopic Heller myotomy with Toupet fundoplication: outcomes predictors in 121 consecutive patients. Archives of surgery (Chicago, Ill : 1960). 2005;140(9):827-833; discussion 833-824.

12 Jeansonne LO, White BC, Pilger KE, et al. Ten-year follow-up of laparoscopic Heller myotomy for achalasia shows durability. Surgical endoscopy. 2007;21(9):1498-1502.

13 Krishnamohan P, Allen MS, Shen KR, et al. Long-term outcome after laparoscopic myotomy for achalasia. The Journal of thoracic and cardiovascular surgery. 2014;147(2):730-736; Discussion 736-737.

14 Perry KA, Kanji A, Drosdeck JM, et al. Efficacy and durability of robotic Heller myotomy for achalasia: patient symptoms and satisfaction at long-term followup. Surgical endoscopy. 2014;28(11):3162-3167.

15 Ruurda JP, Gooszen HG, Broeders IA. Early experience in robot-assisted laparoscopic Heller myotomy. Scandinavian journal of gastroenterology Supplement. 2004(241):4-8.

16 Shaligram A, Unnirevi J, Simorov A, et al. How does the robot affect outcomes? A retrospective review of open, laparoscopic, and robotic Heller myotomy for achalasia. Surgical endoscopy. 2012;26(4):1047-1050.

17 Herbella FA, Armijo PR, Patti MG. A pictorial presentation of 3.0 Chicago Classification for esophageal motility disorders. Einstein (Sao Paulo, Brazil). 2016;14(3):439-442.

18 Gockel I, Junginger T. The value of scoring achalasia: a comparison of current systems and the impact on treatment--the surgeon's viewpoint. The American surgeon. 2007;73(4):327-331.

19 Simorov A, Ranade A, Jones R, et al. Long-term patient outcomes after laparoscopic anti-reflux procedures. Journal of gastrointestinal surgery: official journal of the Society for Surgery of the Alimentary Tract. 2014;18(1):157-162; discussion 162-153.

20 Ross SW, Oommen B, Wormer BA, et al. National outcomes of laparoscopic Heller myotomy: operative complications and risk factors for adverse events. Surgical endoscopy. 2015;29(11):3097-3105.
21 Nau P, Rattner D. Laparoscopic Heller myotomy as the gold standard for treatment of achalasia. Journal of gastrointestinal surgery: official journal of the Society for Surgery of the Alimentary Tract. 2014;18(12):2201-2207.

22 Zonca P, Cambal M, Labas P, et al. The role of laparoscopic Heller myotomy in the treatment of achalasia. Bratislavske lekarske listy. 2014;115(3):156160.

23 Tsuboi K, Omura N, Yano F, et al. Identification of risk factors for mucosal injury during laparoscopic Heller myotomy for achalasia. Surgical endoscopy. 2016;30(2):706-714.

24 Huffmanm LC, Pandalai PK, Boulton BJ, et al. Robotic Heller myotomy: a safe operation with higher postoperative quality-of-life indices. Surgery. 2007;142(4):613-618; discussion 618-620.

25 Diez Del Val I, Martinez Blazquez C, Loureiro Gonzalez C, et al. Robot-assisted gastroesophageal surgery: usefulness and limitations. Journal of robotic surgery. 2014;8(2):111-118.

26 Lynch KL, Pandolfino JE, Howden CW, et al. Major Complications of Pneumatic Dilation and Heller Myotomy for Achalasia: Single Center Experience and Systematic Review of the Literature. The American journal of gastroenterology. 2012;107(12):10.1038/ ajg.2012.1332.

27 Vogel SB, Rout WR, Martin TD, et al. Esophageal perforation in adults: aggressive, conservative treatment lowers morbidity and mortality. Annals of surgery. 2005;241(6):1016-1023.

28 Galvani CA, Gallo AS, Dylewski MR. Roboticassisted Heller myotomy for esophageal achalasia: feasibility, technique, and short-term outcomes. Journal of robotic surgery. 2011;5(3):163-166.

29 Tantau M, Crisan D. Peroral endoscopic myotomy: Time to change our opinion regarding the treatment of achalasia? World journal of gastrointestinal endoscopy. 2015;7(3):237-246.

30 Awaiz A, Yunus RM, Khan S, et al. Systematic Review and Meta-Analysis of Perioperative Outcomes of Peroral Endoscopic Myotomy (POEM) and Laparoscopic Heller Myotomy (LHM) for Achalasia. Surgical laparoscopy, endoscopy \& percutaneous techniques. 2017;27(3):123-131. 
31 Ujiki MB, Yetasook AK, Zapf M, et al. Peroral endoscopic myotomy: A short-term comparison with the standard laparoscopic approach. Surgery. 2013;154(4):893-897; discussion 897-900.

32 Simic AP, Radovanovic NS, Skrobic OM, et al. Significance of limited hiatal dissection in surgery for achalasia. Journal of gastrointestinal surgery: official journal of the Society for Surgery of the Alimentary Tract. 2010;14(4):587-593.

33 Sharata AM, Dunst CM, Pescarus R, et al. Peroral endoscopic myotomy (POEM) for esophageal primary motility disorders: analysis of 100 consecutive patients. Journal of gastrointestinal surgery: official journal of the Society for Surgery of the Alimentary Tract. 2015;19(1):161-170; discussion 170 .

34 Rosemurgy A, Villadolid D, Thometz D, et al. Laparoscopic Heller Myotomy Provides Durable Relief From Achalasia and Salvages Failures After Botox or Dilation. Annals of Surgery. 2005;241(5):725-735.
35 Cacchione RN, Tran DN, Rhoden DH. Laparoscopic Heller myotomy for achalasia. American journal of surgery. 2005;190(2):191-195.

36 Bonatti H, Hinder RA, Klocker J, et al. Long-term results of laparoscopic Heller myotomy with partial fundoplication for the treatment of achalasia. The American Journal of Surgery. 2005;190(6):883-887.

37 Voukelatou P, Vrettos I, Emmanouilidou G, et al. Predictors of Inappropriate Proton Pump Inhibitors Use in Elderly Patients. Current Gerontology and Geriatrics Research. 2019;2019:7591045. 